\title{
Clinical Impact of Intracerebral Hemorrhage after Hyper- acute Extracranial Stenting in Patients with Ischemic Stroke
}

\author{
Je Hong Min, $\mathrm{MD}^{1}$, Seong-Joon Lee, $\mathrm{MD}$, $\mathrm{PhD}^{1}$, Ji Man Hong, $\mathrm{MD}, \mathrm{PhD}^{1}$, Jin Wook Choi, MD, $\mathrm{PhD}^{2}$, \\ Joonsang Yoo, $\mathrm{MD}^{6}$, Chang-Hyun Kim, $\mathrm{MD}^{7}$, Sung-Il Sohn, $\mathrm{MD}, \mathrm{PhD}^{7}$, Yang-Ha Hwang, MD, $\mathrm{PhD}^{5}$, \\ Jin Soo Lee, $\mathrm{MD}, \mathrm{PhD}^{1}$ \\ ${ }^{1}$ Department of Neurology, Ajou University School of Medicine, Ajou University Medical Center, Suwon, Korea \\ ${ }^{2}$ Department of Radiology, Ajou University School of Medicine, Ajou University Medical Center, Suwon, Korea \\ ${ }^{3}$ Department of Neurosurgery, School of Medicine, Kyungpook National University, Daegu, Korea \\ ${ }^{4}$ Department of Radiology, School of Medicine, Kyungpook National University, Daegu, Korea \\ ${ }^{5}$ Department of Neurology, School of Medicine, Kyungpook National University, Daegu, Korea \\ ${ }^{6}$ Department of Neurology, Keimyung University Dongsan Medical Center, Daegu, Korea \\ ${ }^{7}$ Department of Neurosurgery, Keimyung University Dongsan Medical Center, Daegu, Korea
} Dong-Hun Kang, $\mathrm{MD}^{3,4}$, Yong-Won Kim, $\mathrm{MD}^{4,5}$, Yong-Sun Kim, MD, PhD 4 , Jeong-Ho Hong, MD, $\mathrm{PhD}^{6}$,

Purpose: Emergent intracranial occlusions causing acute ischemic stroke are often related to extracranial atherosclerotic stenosis. This study aimed to investigate the association between post-procedure intracerebral hemorrhage $(\mathrm{ICH})$ and emergent extracranial artery stenting and assess their effects on clinical outcomes in patients with acute ischemic stroke.

Materials and Methods: We retrospectively analyzed patients undergoing hyperacute endovascular treatment for cervicocephalic vascular occlusion in three Korean hospitals between January 2011 and February 2016. Patients who had extracranial artery involvement and were treated from 24 hours of symptom onset to puncture were included in this study, and they were divided into the extracranial stenting (ES) and non-ES groups. Any type of petechial hemorrhages and parenchymal hematoma was defined as $\mathrm{ICH}$ for the current study.

Results: In total, 76 patients were included in this study. Among them, 56 patients underwent ES, and 20 patients did not. Baseline characteristics, risk factors, laboratory data, treatment methods, successful reperfusion rates, and baseline stenotic degrees of extracranial internal carotid artery did not differ between these two groups. However, atrial fibrillation was more frequent in patients without than with $\mathrm{ES}(\mathrm{P}=0.002)$, and post-procedure $\mathrm{ICH}$ was more frequent in patients with than without $E S(P=0.035)$. Logistic regression models revealed that $E S$ was independently associated with post-procedure ICH (odds ratio [OR], 7.807; 95\% confidence interval $[\mathrm{Cl}], 1.213-50.248 ; \mathrm{P}=0.031)$, and ICH was independently associated with poor clinical outcomes (OR, 0.202; 95\% Cl, 0.054-0.759; $P=0.018)$; however, ES itself was not associated with clinical outcomes (OR, 0.530; 95\% Cl, 0.117-2.395; $\mathrm{P}=0.409)$. Notably, ICH and ES had interaction for predicting good outcomes $(\mathrm{P}=0.041)$.

Conclusion: Post-procedure ICH was associated with ES and poor clinical outcomes. Therefore, ES should be cautiously considered in patients with hyperacute stroke.

Key Words: Carotid stenosis; Stents; Cerebral infarction; Endovascular procedures; Cerebral hemorrhage

\author{
Correspondence to: \\ Jin Soo Lee, MD, PhD \\ Department of Neurology, Ajou Uni- \\ versity School of Medicine, 164 World \\ Cup-ro, Yeongtong-gu, Suwon 16499, \\ Korea \\ Tel: +82-31-219-5175 \\ Fax: +82-31-219-5178 \\ E-mail:jinsoo22@gmail.com
}

Received: May 31, 2019

Revised: June 26, 2019

Accepted: June 27, 2019

Copyright $\odot 2019$ Korean Society of Interventional Neuroradiology This is an Open Access article distributed under the terms of the Creative Commons Attribution Non-Commercial License (http://creativecommons.org/licenses/by-nc/3.0) which permits unrestricted non-commercial use, distribution, and reproduction in any medium, provided the original work is properly cited.

pISSN 2093-9043 eISSN 2233-6273 


\section{INTRODUCTION}

Extracranial atherosclerosis (ECAS) is observed in about 10$20 \%$ of stroke patients and is not a rare cause of acute ischemic stroke. ${ }^{1,2}$ Retrospective single-arm studies have shown that acute ischemic stroke patients undergoing emergent endovascular treatment (EVT) for acute extracranial internal carotid artery (ICA) occlusion show high recanalization rates and early neurologic improvement. ${ }^{3-5}$ In addition, several studies have demonstrated that patients with extracranial occlusion combined with intracranial artery tandem occlusion have better outcomes after EVT. ${ }^{6-8}$ Mechanical thrombectomy-based EVT is now considered a reasonable treatment especially for intracranial large artery occlusions. ${ }^{9}$ However, symptomatic intracranial hemorrhage $(\mathrm{ICH})$ is observed in about 30-40\% patients with acute large-artery occlusion after $\mathrm{EVT}^{10,11}$ and it is thus considered a significant contributing factor for poor functional outcomes.

Carotid artery stenting is an EVT option for patients with extracranial carotid stenosis. Cases of extracranial stenting (ES) for ECAS-related occlusion in hyperacute stroke are increasing presently; however, the relationship between ES and the prevalence of post-procedural hemorrhagic transformation $(H T)$ remains unclear. Therefore, we investigated the association between ES and the prevalence of post-procedural HT and assessed the effect of ES on clinical outcomes in patients with hyperacute stroke.

\section{MATERIALS AND METHODS}

\section{Study population and inclusion criteria}

We retrospectively analyzed patients in the Acute Stroke due to Intracranial Atherosclerotic occlusion and Neurointervention-Korean Retrospective (ASIAN-KR) registry. This registry collected data on patients (aged $\geq 18$ years) who underwent EVT for cervicocerebral artery occlusion causing ischemic stroke at three comprehensive stroke centers in Korea between January 2011 and February 2016. ${ }^{12}$ Inclusion criteria were as follows: 1) onset-to-puncture time within 24 hours; 2) underlying etiology was proven as ECAS; or 3) patients underwent EVT for extracranial artery steno-occlusive disease.

All clinical data were de-identified and allocated study identification numbers. The protocol of data collection was approved by the Institutional Review Board of each hospital and was implemented in accordance with the ethical stan- dards of the 1964 Declaration of Helsinki and its later amendments.

\section{Evaluation methods}

Premorbid and 3-month modified Rankin Scale (mRS) scores, National Institutes of Health Stroke Scale (NIHSS) scores, conventional vascular risk factors, and laboratory findings on admission were collected. A 3-month mRS score of 0 to 2 or no change compared with the premorbid mRS score was defined as a good outcome, and a 3-month mRS score of 3 to 6 or an increase compared with the premorbid mRS score was classified as a poor outcome.

The location of the initial occlusion site was determined using baseline computed tomography (CT) angiography, magnetic resonance angiography, or digital subtraction angiography. Stenotic degree of extracranial artery associated with hyperacute stroke was assessed based on the North American Symptomatic Carotid Endarterectomy Trial criteria. ${ }^{13}$ Alberta Stroke Program Early CT Scores (ASPECTS) were determined using non-contrast $\mathrm{CT}$ in the anterior-circulation territory in patients with hyperacute stroke. Final reperfusion was evaluated using the modified thrombolysis in cerebral infarction (mTICl) scale, ${ }^{14}$ and $\mathrm{mTICl}$ grades $2 \mathrm{~b}$ or 3 were defined as successful reperfusion. Post-procedural ICH was graded based on the criteria defined by the European Cooperative Acute Stroke Study, and was defined as presence of any grade of $\mathrm{ICH}$ for the current study. ${ }^{15}$ Subarachnoid hemorrhage was classified according to the modified Fisher scale. ${ }^{16}$

\section{Endovascular procedures}

Endovascular devices were selected at the discretion of neurointerventionists based on consensus within each stroke team. Contact aspiration and stent retrieval were routinely used as a frontline method. Contact aspiration is a method of a forced arterial suction thrombectomy, which uses the Penumbra system (Penumbra Inc., Alameda, CA, USA). Stent retrieval is a method of clot removal by capturing and removing the thrombus with a stent retriever, such as the Solitaire AB/FR (Medtronic, Irvine, CA, USA) or Trevo (Stryker, Kalamazoo, MI, USA). Balloon guide catheters, adjuvant local lytic infusion, intracranial or extracranial angioplasty, and/or stenting were implemented as needed.

\section{Statistical analysis}

Data are expressed as mean \pm standard deviations, median 
(interquartile ranges), or numbers (percentages). Comparative analyses of clinical characteristics and treatment outcomes were performed between the ES and non-ES (NES) groups. Differences between the groups were analyzed using $x^{2}$ tests for categorical variables, the Mann-Whitney U-test for ordinal variables, or t-tests for continuous variables. To evaluate the relation between ES and the prevalence of post-procedural $\mathrm{ICH}$ or further clinical outcomes, we performed multivariate logistic regression analyses with major confounders. A P of $<0.05$ was considered significant. Statistical analyses were performed using the SPSS statistical package (version 25.0, IBM SPSS, Armonk, NY, USA).

\section{RESULTS}

\section{Baseline characteristics}

Among the 720 registered patients, 76 patients were eligible based on the inclusion criteria (Fig. 1). Baseline characteristics of patients are summarized in Table 1. Among the 76 eligible patients, 56 patients underwent ES, and 20 patients did not undergo any extracranial treatment or underwent other extracranial treatments without stenting (such as balloon-angioplasty and contract aspiration). Atrial fibrillation was more prevalent in the NES group than in the ES group (35.0\% vs.
$7.1 \%, P=0.002) ;$ however, other variables including laboratory data; percentages of hypertension, diabetes mellitus, dyslipidemia, or smoker; NIHSS or ASPECTS score on admission; or systolic or diastolic blood pressure did not differ between the two groups.

\section{Treatment methods and outcomes associated with ES}

Comparative analyses of treatment methods and outcomes between these two groups are summarized in Table 2. Overall procedure time or treatment methods, except for the $E S$, were not different between the two groups. The rates of successful reperfusion were similar in these two groups, regardless of ES ( $80.0 \%$ vs. $85.7 \%, \mathrm{P}=0.547)$. Functional outcomes, based on the mRS score, did not differ between the ES and NES groups. However, the prevalence of post-procedural cerebral hemorrhage after emergent extracranial EVT was associated with ES (odds ratio [OR], 7.807; 95\% confidence interval [CI], 1.213 to 50.248; $P=0.031$ ) (Table 3). Notably, multivariate analyses with potential major confounders in a logistic regression model revealed that the presence of ES was the only independent predictor for the prevalence of hemorrhage after emergent extracranial EVT (OR, 7.807; 95\% $\mathrm{Cl}, 1.213$ to $50.248 ; \mathrm{P}=0.031$ ). Good outcomes at 3 months were more commonly observed in patients without than

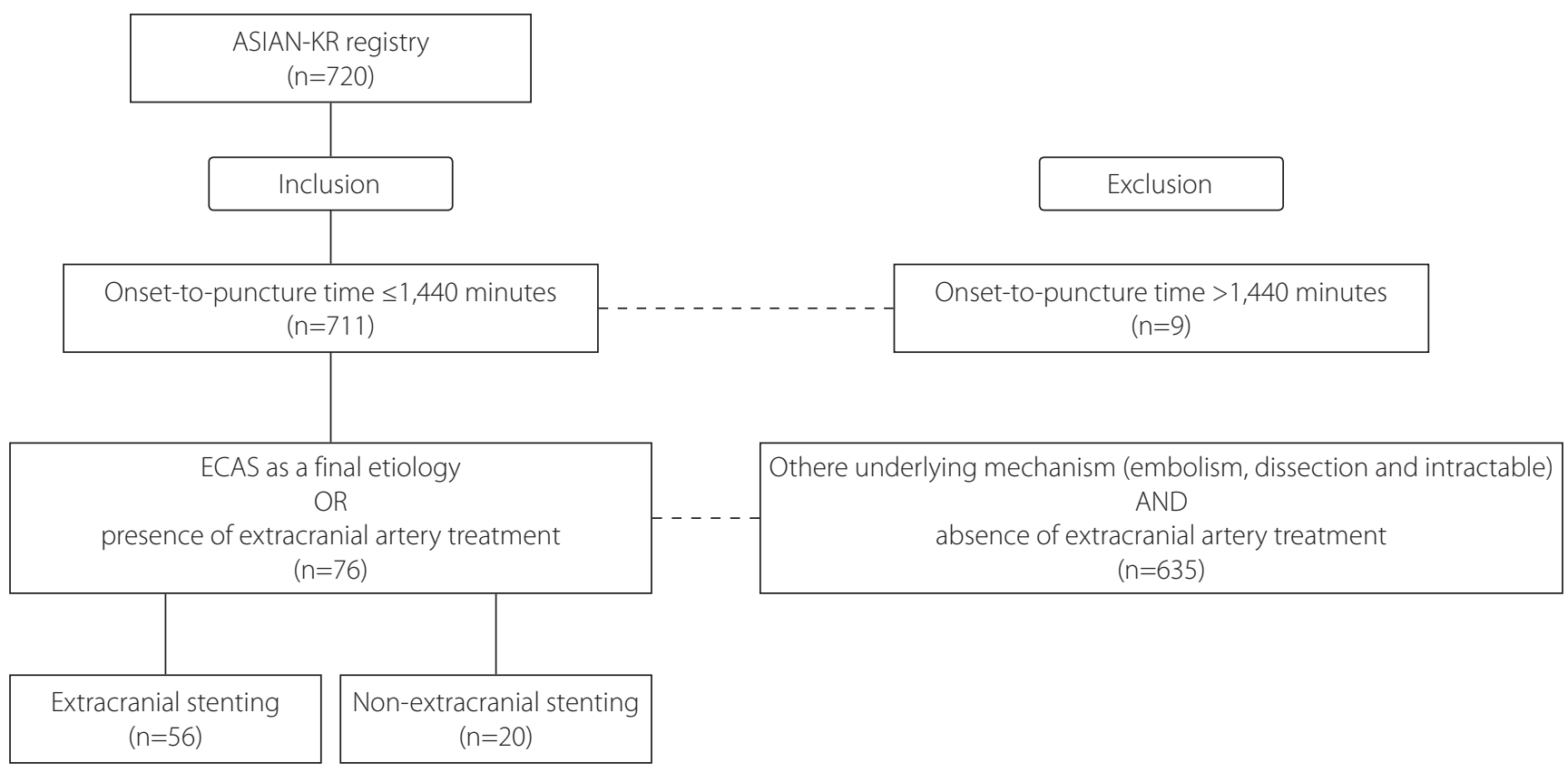

Fig. 1. Flowchart of this study. ASIAN-KR, Acute Stroke due to Intracranial Atherosclerotic occlusion and Neurointervention-Korean Retrospective; ECAS, Extracranial Atherosclerosis. 
Table 1. Baseline characteristics of patients with emergent extracranial steno-occlusive disease

\begin{tabular}{|c|c|c|c|}
\hline & No extracranial stenting $(n=20)$ & Extracranial stenting $(n=56)$ & P-value \\
\hline Age & $67.9 \pm 7.7$ & $69.1 \pm 8.8$ & 0.588 \\
\hline Sex, men & $16(80.0)$ & $49(87.5)$ & 0.413 \\
\hline Hypertension & $13(65.0)$ & $38(67.9)$ & 0.815 \\
\hline Diabetes mellitus & $5(25.0)$ & $21(37.5)$ & 0.312 \\
\hline Dyslipidemia & $7(35.0)$ & $17(30.4)$ & 0.701 \\
\hline Atrial fibrillation & $7(35.0)$ & $4(7.1)$ & 0.002 \\
\hline Smoker & $6(30.0)$ & $21(37.5)$ & 0.547 \\
\hline Intracranial occlusion sites & & & 0.620 \\
\hline ICA, T-type & $11(55.0)$ & $29(51.8)$ & \\
\hline MCA, M1 & $7(35.0)$ & $21(37.5)$ & \\
\hline MCA, M2 & $0(0.0)$ & $1(1.8)$ & \\
\hline Vertebrobasilar artery & $2(10.0)$ & $2(3.6)$ & \\
\hline None, extracranial ICA only & $0(0.0)$ & $3(5.4)$ & \\
\hline Initial NIHSS score, median & $16(10.5-21)$ & $14(10-19)$ & 0.469 \\
\hline ASPECTS score, median & $7(6-8)(n=15)$ & $7(5.8-9)(n=50)$ & 0.534 \\
\hline Hemoglobin (g/dL) & $13.3 \pm 1.2$ & $13.7 \pm 1.6$ & 0.275 \\
\hline White blood cell $\left(\times 10^{9} / \mathrm{L}\right)$ & $9.8 \pm 3.2$ & $9.8 \pm 3.3$ & 0.958 \\
\hline Platelet $\left(\times 10^{9} / \mathrm{L}\right)$ & $253.2 \pm 111.4$ & $254.7 \pm 65.2$ & 0.942 \\
\hline Glucose (mg/dL) & $124.3 \pm 34.8$ & $144.0 \pm 65.5$ & 0.097 \\
\hline Total cholesterol (mg/dL) & $169.0 \pm 45.0$ & $173.8 \pm 46.9$ & 0.692 \\
\hline Low-density lipoprotein (mg/dL) & $111.5 \pm 40.4$ & $108.6 \pm 43.7$ & 0.792 \\
\hline High-density lipoprotein (mg/dL) & $41.4 \pm 7.8$ & $41.4 \pm 10.2$ & 0.994 \\
\hline Triglyceride (mg/dL) & $112.7 \pm 80.3$ & $147.9 \pm 217.8$ & 0.484 \\
\hline Erythrocyte sedimentation rate (mm/hr) & $19.6 \pm 20.9$ & $18.6 \pm 14.3$ & 0.815 \\
\hline C-reactive protein $(\mathrm{mg} / \mathrm{dL})$ & $2.0 \pm 4.3$ & $1.0 \pm 2.4$ & 0.316 \\
\hline Initial systolic blood pressure (mmHg) & $162.2 \pm 29.0$ & $157.8 \pm 27.7$ & 0.551 \\
\hline Initial diastolic blood pressure (mmHg) & $85.5 \pm 18.4$ & $85.9 \pm 14.1$ & 0.905 \\
\hline
\end{tabular}

Values are presented as mean \pm standard deviation or number (\%).

ICA, internal carotid artery; MCA, middle cerebral artery; NIHSS, National Institutes of Health Stroke Scale; ASPECTS, Alberta Stroke Program Early CT Score.

with any post-procedural cerebral hemorrhage (68.0\% vs. 42.3\%, $\mathrm{P}=0.031$ ).

As observed in Table 4, the association between ES and better clinical outcomes was not demonstrated in multivariate logistic regression analyses (OR, 0.530; $95 \% \mathrm{Cl}, 0.117$ to 2.395; $P=0.409$ ). However, good outcomes after EVT were the absence of any post-procedural intracerebral hemorrhage (OR, 0.202; 95\% Cl, 0.054 to 0.759; $\mathrm{P}=0.018$ ). Because ES had an interaction with post-procedural intracerebral hemorrhage $(P=0.041)$, we speculate that $E S$ has an indirect impact on worse clinical outcomes.

\section{DISCUSSION}

In the present study, we found that the prevalence of post-procedural ICH was relatively higher in patients who underwent emergent ES than in those who did not. Good outcomes after hyperacute extracranial artery treatment were attributable to multiple factors such as younger age, lower initial NIHSS score, successful reperfusion, and the absence of any ICH after EVT. Post-procedural ICH was related emergent extracranial artery stenting and associated with poor functional outcomes. 
Table 2. Comparisons of treatment methods and outcomes

\begin{tabular}{|c|c|c|c|}
\hline & No extracranial stenting $(n=20)$ & Extracranial stenting $(n=56)$ & P-value \\
\hline IV thrombolysis & $9(45.0)$ & $25(44.6)$ & 0.978 \\
\hline Onset to puncture time (minutes) & $448.5 \pm 351.0$ & $384.1 \pm 245.8$ & 0.375 \\
\hline Procedure time (minutes) & $78.1 \pm 58.7$ & $78.8 \pm 41.0$ & 0.948 \\
\hline Use of balloon guide catheter & $6(30.0)$ & $17(30.4)$ & 0.976 \\
\hline Remote aspiration with balloon guide catheter & $14(70.0)$ & $36(64.3)$ & 0.644 \\
\hline Stent retrieval & $6(30.0)$ & $17(30.4)$ & 0.976 \\
\hline Contact aspiration & $16(80.0)$ & $37(66.1)$ & 0.244 \\
\hline Local tirofiban infusion & $4(20.0)$ & $7(12.5)$ & 0.413 \\
\hline Final reperfusion success & $16(80.0)$ & $48(85.7)$ & 0.547 \\
\hline Post-procedure intracerebral hemorrhage & & & 0.160 \\
\hline None & $17(85.0)$ & $33(58.9)$ & \\
\hline Hemorrhagic infarct type 1 & $0(0.0)$ & $6(10.7)$ & \\
\hline Hemorrhagic infarct type 2 & $1(5.0)$ & $10(17.9)$ & \\
\hline Parenchymal hematoma type 1 & $0(0.0)$ & $3(5.4)$ & \\
\hline Parenchymal hematoma type 2 & $2(10.0)$ & $4(7.1)$ & \\
\hline Post-procedure subarachnoid hemorrhage & & & 0.646 \\
\hline None & $19(95.0)$ & $52(92.9)$ & \\
\hline Fisher grade 1 & $0(0.0)$ & $1(1.8)$ & \\
\hline Fisher grade 2 & $0(0.0)$ & $2(3.6)$ & \\
\hline Fisher grade 3 & $0(0.0)$ & $0(0.0)$ & \\
\hline Fisher grade 4 & $1(5.0)$ & $1(1.8)$ & \\
\hline Modified Rankin Scale, median & $2(1-3)$ & $2(1-4)$ & 0.516 \\
\hline
\end{tabular}

Values are presented as mean \pm standard deviation or number (\%).

IV, intravenous.

It is well known that EVTs such as mechanical thrombectomy are preferred to intravenous thrombolysis for intracranial large artery occlusions. ${ }^{9,17}$ Whereas EVTs for intracranial large artery occlusions have had sufficient evidences of efficacy and safety, evidences for emergent ES have barely been achieved. Nevertheless, accumulating evidence has indicated the safety and efficacy of emergent endovascular stenting in hyperacute stroke. It was reported that endovascular therapy of tandem occlusions with extracranial internal carotid artery revascularization as the first step had a high recanalization rate and led to an acceptable rate of good clinical outcomes. However, little is known about the hemorrhagic risk after ES. A retrospective study about the hemorrhagic risk after emergent EVT with intracranial or ES showed that patients undergoing emergent stenting had higher grades of hemorrhagic subtypes, and acute phase stenting was an independent predictor of $\mathrm{sICH}$ after adjustment for potential confound- ers and the procedure duration. However, this study had a single-center retrospective design, and the sample size was small (24 patients) in the emergent stenting group. ${ }^{18}$ Notably, TITAN study did not find a definite association between extracranial ICA stenting and post-procedural hemorrhage, although procedure-related complications after 90 days of $E V T$, including any $\mathrm{ICH}$, were lower in the intracranial thrombectomy with ICA stenting group than in the intracranial thrombectomy only group. ${ }^{19}$ However, while final successful reperfusion rate was similar between ES and NES groups in the current study, reperfusion success rate was significantly higher in the ICA stenting group than thrombectomy only group in the TITAN study. Reperfusion failure may increase the final infarct volume, which, in turn, can cause larger area of breakdown of blood-brain barrier and the risk of hemorrhagic transformation. Therefore, it is difficult to ascertain that ES-based EVT is safer than NES-based. 
Table 3. Risk factors of intracerebral hemorrhage after EVT

\begin{tabular}{|c|c|c|c|c|c|}
\hline & \multicolumn{3}{|c|}{ Univariate analysis } & \multicolumn{2}{|c|}{ Multivariate analysis } \\
\hline & No hemorrhage $(n=50)$ & Any hemorrhage $(n=26)$ & P-value & OR $(95 \% \mathrm{Cl})$ & P-value \\
\hline Age & $68.7 \pm 8.2$ & $68.9 \pm 9.2$ & 0.944 & $1.018(0.947-1.094)$ & 0.623 \\
\hline Sex, men & $42(84.0)$ & $23(88.5)$ & 0.600 & $1.139(0.165-7.878)$ & 0.895 \\
\hline Hypertension & $33(70.2)$ & $17(68.0)$ & 0.846 & & \\
\hline Diabetes mellitus & $19(40.4)$ & $7(28.0)$ & 0.296 & $0.657(0.149-2.896)$ & 0.579 \\
\hline Atrial fibrillation & $7(14.9)$ & $2(8.0)$ & 0.400 & & \\
\hline Smoker & $15(31.9)$ & $10(40.0)$ & 0.493 & & \\
\hline Dyslipidemia & $16(34.0)$ & $8(32.0)$ & 0.861 & & \\
\hline Prior history of TIA or stroke & $7(14.9)$ & $4(16.0)$ & 0.901 & $3.083(0.402-23.658)$ & 0.279 \\
\hline Prior usage of antiplatelets & $12(25.5)$ & $7(28.0)$ & 0.821 & $1.199(0.263-5.466)$ & 0.815 \\
\hline Prior usage of anticoagulants & $1(2.1)$ & $1(4.0)$ & 0.645 & $15.776(0.084-2975.334)$ & 0.302 \\
\hline Initial NIHSS score, median & $14(10-19.3)$ & $15.5(10.8-20.3)$ & 0.781 & $0.999(0.904-1.104)$ & 0.984 \\
\hline Intracranial occlusion location & & & 0.405 & & \\
\hline ICA, T-type & $27(54.0)$ & $13(50.0)$ & & & \\
\hline MCA, M1 & $17(34.0)$ & $11(42.3)$ & & & \\
\hline$M C A, M 2$ & $0(0.0)$ & $1(3.8)$ & & & \\
\hline Vertebrobasilar artery & $3(6.0)$ & $0(0.0)$ & & & \\
\hline None, only extracranial ICA & $3(6.0)$ & $0(0.0)$ & & & \\
\hline ASPECTS score, median & $8(5.5-9)(n=41)$ & $6(6-8)(n=24)$ & 0.071 & & \\
\hline Hemoglobin (g/dL) & $13.3 \pm 1.6$ & $14.1 \pm 1.2$ & 0.036 & & \\
\hline White blood cell $\left(\times 10^{9} / \mathrm{L}\right)$ & $9.6 \pm 3.2$ & $10.3 \pm 3.3$ & 0.372 & & \\
\hline Platelet $\left(\times 10^{9} / \mathrm{L}\right)$ & $253.7 \pm 88.5$ & $255.6 \pm 58.4$ & 0.921 & & \\
\hline Glucose (mg/dL) & $135.2 \pm 44.4$ & $145.7 \pm 81.5$ & 0.545 & & \\
\hline Total cholesterol (mg/dL) & $163.4 \pm 45.0$ & $190.0 \pm 44.0$ & 0.016 & & \\
\hline Low-density lipoprotein (mg/dL) & $101.6 \pm 40.0$ & $124.0 \pm 44.8$ & 0.029 & & \\
\hline High-density lipoprotein (mg/dL) & $39.1 \pm 9.1$ & $45.8 \pm 9.1$ & 0.003 & & \\
\hline Triglyceride (mg/dL) & $149.0 \pm 231.3$ & $118.7 \pm 68.8$ & 0.517 & & \\
\hline Initial systolic blood pressure (mmHg) & $160.2 \pm 28.4$ & $156.6 \pm 27.3$ & 0.593 & & \\
\hline Initial diastolic blood pressure (mmHg) & $86.9 \pm 14.2$ & $83.6 \pm 17.1$ & 0.370 & & \\
\hline IV thrombolysis & $23(46.0)$ & $11(42.3)$ & 0.759 & $1.735(0.327-9.199)$ & 0.518 \\
\hline Onset to puncture time (minutes) & $388.0 \pm 270.5$ & $426.1 \pm 290.8$ & 0.571 & $1.001(0.998-1.004)$ & 0.414 \\
\hline Procedure time (minutes) & $81.1 \pm 45.6$ & $73.8 \pm 46.9$ & 0.512 & $0.995(0.980-1.010)$ & 0.516 \\
\hline Use of balloon guide catheter & $33(66.0)$ & $17(65.4)$ & 0.957 & & \\
\hline $\begin{array}{l}\text { Remote aspiration with balloon guide } \\
\text { catheter }\end{array}$ & $17(34.0)$ & $6(23.1)$ & 0.325 & & \\
\hline Stent retrieval & $12(24.0)$ & $11(42.3)$ & 0.099 & & \\
\hline Contact aspiration & $35(70.0)$ & $18(69.2)$ & 0.945 & & \\
\hline Local tirofiban infusion & $5(10.0)$ & $6(23.1)$ & 0.124 & & \\
\hline Extracranial stenting & $33(66.0)$ & $23(88.5)$ & 0.035 & $7.807(1.213-50.248)$ & 0.031 \\
\hline Successful reperfusion & $42(84.0)$ & $22(84.6)$ & 0.944 & & \\
\hline Good outcomes at 3 months & $34(68.0)$ & $11(42.3)$ & 0.031 & & \\
\hline
\end{tabular}

Values are presented as mean \pm standard deviation or number (\%) unless otherwise indicated.

EVT, endovascular treatment; OR, odds ratio; Cl, confidence interval; TIA, transient ischemic attack; NIHSS, National Institutes of Health Stroke Scale; ICA, internal carotid artery; MCA, middle cerebral artery; ASPECTS, Alberta Stroke Program Early CT Score; IV, intravenous. 
Table 4. Predicting factors associated with good clinical outcomes

\begin{tabular}{|c|c|c|c|c|c|}
\hline & & riate analysis & & Multivariate ana & ysis \\
\hline & $\begin{array}{l}\text { Poor outcomes } \\
\quad(n=31)\end{array}$ & $\begin{array}{l}\text { Good outcomes } \\
\qquad(n=45)\end{array}$ & P-value & OR $(95 \% \mathrm{Cl})$ & P-value \\
\hline Age & $71.9 \pm 9.5$ & $66.6 \pm 7.0$ & 0.007 & $0.918(0.848-0.993)$ & 0.033 \\
\hline Sex, men & $27(87.1)$ & $38(84.4)$ & 0.747 & $0.841(0.162-4.353)$ & 0.837 \\
\hline Hypertension & $22(71.0)$ & $29(64.4)$ & 0.552 & & \\
\hline Diabetes mellitus & $10(32.3)$ & $16(35.6)$ & 0.766 & $0.687(0.177-2.665)$ & 0.587 \\
\hline Atrial fibrillation & 7 (22.6) & $4(8.9)$ & 0.095 & $0.216(0.027-1.718)$ & 0.147 \\
\hline Smoker & $7(22.6)$ & $20(44.4)$ & 0.050 & & \\
\hline Dyslipidemia & $8(25.8)$ & $16(35.6)$ & 0.369 & & \\
\hline Premorbid mRS score, median & $0(0-1)$ & $0(0-0)$ & 0.635 & & \\
\hline Initial NIHSS score, median & $19(11-22)$ & $14(10-17)$ & 0.126 & $0.895(0.801-0.999)$ & 0.049 \\
\hline Initial occlusion site & & & 0.453 & & \\
\hline ICA, T-type & $15(48.4)$ & $25(55.6)$ & & & \\
\hline MCA, M1 & $11(35.5)$ & $17(37.8)$ & & & \\
\hline $\mathrm{MCA}, \mathrm{M} 2$ & $1(3.2)$ & $0(0.0)$ & & & \\
\hline Vertebrobasilar artery & $3(9.7)$ & $1(2.2)$ & & & \\
\hline None, only extracranial ICA & $1(3.2)$ & $2(4.4)$ & & & \\
\hline ASPECTS score, median & $6.5(4.3-8.8)(n=24)$ & $6(7-9)(n=41)$ & 0.610 & & \\
\hline Onset to puncture time (minutes) & $461.3 \pm 332.8$ & $359.5 \pm 224.2$ & 0.144 & $0.998(0.996-1.000)$ & 0.117 \\
\hline Puncture to final time (minutes) & $89.7 \pm 55.2$ & $71.0 \pm 36.9$ & 0.104 & & \\
\hline Prior history of TIA or stroke & $5(16.1)$ & $8(17.8)$ & 0.851 & & \\
\hline Prior usage of antiplatelets & $6(19.4)$ & $14(31.1)$ & 0.253 & & \\
\hline Prior usage of anticoagulants & $1(3.2)$ & $2(4.4)$ & 0.789 & & \\
\hline Initial systolic blood pressure (mmHg) & $156.0 \pm 26.1$ & $161.0 \pm 29.2$ & 0.450 & & \\
\hline Initial diastolic blood pressure $(\mathrm{mmHg})$ & $85.6 \pm 12.6$ & $85.9 \pm 16.9$ & 0.929 & & \\
\hline Hemoglobin (g/dL) & $13.4 \pm 1.6$ & $13.7 \pm 1.4$ & 0.354 & & \\
\hline White blood cell $\left(\times 10^{9} / \mathrm{L}\right)$ & $10.6 \pm 3.6$ & $9.3 \pm 2.9$ & 0.090 & & \\
\hline Platelet $\left(\times 10^{9} / \mathrm{L}\right)$ & $234.8 \pm 69.4$ & $267.7 \pm 83.3$ & 0.075 & & \\
\hline Glucose (mg/dL) & $143.3 \pm 73.9$ & $135.7 \pm 47.7$ & 0.586 & & \\
\hline Total cholesterol (mg/dL) & $174.5 \pm 46.9$ & $171.1 \pm 46.1$ & 0.753 & & \\
\hline Low-density lipoprotein (mg/dL) & $111.0 \pm 46.6$ & $108.3 \pm 40.3$ & 0.787 & & \\
\hline High-density lipoprotein (mg/dL) & $43.0 \pm 10.2$ & $40.4 \pm 9.1$ & 0.255 & & \\
\hline Triglyceride (mg/dL) & $152.7 \pm 286.9$ & $129.1 \pm 83.3$ & 0.604 & & \\
\hline IV thrombolysis & $11(35.5)$ & $23(51.1)$ & 0.178 & $1.059(0.236-4.752)$ & 0.940 \\
\hline Use of balloon guide catheter & $17(54.8)$ & $33(73.3)$ & 0.095 & & \\
\hline $\begin{array}{l}\text { Remote aspiration with balloon guide } \\
\text { catheter }\end{array}$ & 7 (22.6) & 16 (35.6) & 0.226 & & \\
\hline Stent retrieval & $14(45.2)$ & $9(20.0)$ & 0.019 & & \\
\hline Contact aspiration & $19(61.3)$ & $34(75.6)$ & 0.183 & & \\
\hline Local tirofiban infusion & $4(12.9)$ & $7(15.6)$ & 0.747 & & \\
\hline Extracranial stenting* & $23(74.2)$ & $33(73.3)$ & 0.933 & $0.530(0.117-2.395)$ & 0.409 \\
\hline Successful reperfusion & $23(74.2)$ & $41(91.1)$ & 0.047 & $13.892(2.132-90.538)$ & 0.006 \\
\hline $\begin{array}{l}\text { Post-procedure intracerebral } \\
\text { hemorrhage, any type* }\end{array}$ & $15(48.4)$ & $11(24.4)$ & 0.037 & $0.202(0.054-0.759)$ & 0.018 \\
\hline $\begin{array}{l}\text { Post-procedure subarachnoid } \\
\text { hemorrhage, any type }\end{array}$ & $3(9.7)$ & $2(4.4)$ & 0.294 & & \\
\hline
\end{tabular}

Values are presented as mean \pm standard deviation or number (\%) unless otherwise indicated.

OR, odds ratio; Cl, confidence interval; mRS: modified Rankin Scale; NIHSS; National Institutes of Health Stroke Scale; ICA, internal carotid artery; MCA, middle cerebral artery; ASPECTS, Alberta Stroke Program Early CT Score; TIA, transient ischemic attack; IV, intravenous.

*Both variables had interaction ( $P=0.041)$. 
Several reasons may explain the relative high risk of $\mathrm{ICH}$ following ES in the current study. Many studies have suggested that hyperacute $\mathrm{ICH}$ after extracranial carotid artery stenting is mainly associated with abrupt cerebral hyperperfusion in the chronically hypoperfused areas. ${ }^{20-23}$ The high rate of cerebral hyperperfusion after stenting is associated with the underlying diseases causing microangiopathy, high degrees of stenosis with the poor collateral flow, high degrees of stenosis on the contralateral carotid artery, and recent stroke history. ${ }^{20,21}$ In addition, these post-procedural hemorrhages just after extracranial carotid artery stenting could result from rupture of small perforating arteries after rapidly normalized perfusion pressure in the chronically hypoperfused areas. ${ }^{24}$ Prior usage of antiplatelets or anticoagulants might also increase the risk of post-procedural hemorrhage. In our current study, no significant correlation was observed between prior antiplatelet/anticoagulant medication and post-procedural hemorrhage. However, peri- or post-procedural antiplatelet treatment might potentially affect the incidence of $\mathrm{ICH}$ in the current study. As large infarct volume or microbleeds are seen on $\mathrm{CT}$ or magnetic resonance imaging, physicians may skip antiplatelet medication to escape hemorrhagic transformation. In contrast, physicians cannot skip the medication if ES is permanently deployed to escape in-stent thrombosis. Therefore, the occurrence of ICH after stenting might be caused by sudden hyperemia/hyperperfusion combined with post-stent prevention, such as antiplatelets.

Many studies have improved the process of EVT for intracranial large artery occlusion (LAO) in patients with acute ischemic stroke and tandem occlusion from ECAS disease. It is suggested that sufficient dilation of stenosis cervical artery is important for the successful use in stent-retrieval thrombectomy or spontaneous recanalization in cases of refractory $\mathrm{LAO}^{25}$ Therefore, it is necessary to perform balloon angioplasty or stenting for maintaining a good extracranial artery perfusion status. Our study showed that ES had an increased risk on post-procedure $\mathrm{ICH}$; therefore, balloon angioplasty could be used as an alternative method, and cautious local administration of glycoprotein IIb/Illa inhibitor, such as tirofiban, could be used to prevent re-occlusion of the extracranial artery after mechanical thrombectomy.

This study has several limitations. First, this study was a retrospective study; thus, the findings in the current study cannot be generalized. For escaping potential biases, we recruited multicenter data. Second, ICH after ES in patients with posterior circulation stroke was not well evaluated. Therefore, further studies must be implemented with a large amount of data related to extracranial vertebral artery stenting. Third, no exact data on pre- or post-stenting prevention were available; therefore, we could not exactly examine whether post-procedural ICH was related to stenting or/and antiplatelet medication during the stenting period. Therefore, further studies with a standardized dosage of antiplatelet medications before and after stenting are needed.

\section{CONCLUSION}

Emergent extracranial artery stenting has been used as a treatment option for hyperacute stroke patients with extracranial artery stenosis. This study found that ICH more frequently occurred in patients who underwent ES and was related to poor clinical outcomes in which ICH and ES had interaction. The findings indicate that ES must be cautiously considered in patients with ECAS-related acute ischemic stroke.

\section{REFERENCES}

1. Grau AJ, Weimar C, Buggle F, Heinrich A, Goertler M, Neumaier S, et al. Risk factors, outcome, and treatment in subtypes of ischemic stroke: the German stroke data bank. Stroke 2001;32:25592566

2. Rubiera M, Ribo M, Delgado-Mederos R, Santamarina E, Delgado P, Montaner J, et al. Tandem internal carotid artery/middle cerebral artery occlusion: an independent predictor of poor outcome after systemic thrombolysis. Stroke 2006;37:2301-2305

3. Jovin TG, Gupta R, Uchino K, Jungreis CA, Wechsler LR, Hammer $M D$, et al. Emergent stenting of extracranial internal carotid artery occlusion in acute stroke has a high revascularization rate. Stroke 2005;36:2426-2430

4. Papanagiotou P, Roth C, Walter S, Behnke S, Grunwald IQ, Viera $\mathrm{J}$, et al. Carotid artery stenting in acute stroke. J Am Coll Cardiol 2011;58:2363-2369

5. Yoon W, Kim BM, Kim DJ, Kim DI, Kim SK. Outcomes and prognostic factors after emergent carotid artery stenting for hyperacute stroke within 6 hours of symptom onset. Neurosurgery 2015;76:321-329

6. Grigoryan M, Haussen DC, Hassan AE, Lima A, Grossberg J, Rebello LC, et al. Endovascular treatment of acute ischemic stroke due to tandem occlusions: large multicenter series and system- 
atic review. Cerebrovasc Dis 2016;41:306-312

7. Maurer C, Joachimski F, Berlis A. Two in one: endovascular treatment of acute tandem occlusions in the anterior circulation. Clin Neuroradiol 2015;25:397-402

8. Cohen JE, Gomori JM, Rajz G, Itshayek E, Eichel R, Leker RR. Extracranial carotid artery stenting followed by intracranial stentbased thrombectomy for acute tandem occlusive disease. $J$ Neurointerv Surg 2015;7:412-417

9. Powers WJ, Rabinstein AA, Ackerson T, Adeoye OM, Bambakidis NC, Becker K, et al. 2018 guidelines for the early management of patients with acute ischemic stroke: a guideline for healthcare professionals from the American Heart Association/American Stroke Association. Stroke 2018;49:e46-e99.

10. Kang DH, Yoon W, Kim SK, Baek BH, Lee YY, Kim YW, et al. Endovascular treatment for emergent large vessel occlusion due to severe intracranial atherosclerotic stenosis. J Neurosurg 2019;130:1949-1956

11. Hao Y, Yang D, Wang H, Zi W, Zhang M, Geng Y, et al. Predictors for symptomatic intracranial hemorrhage after endovascular treatment of acute ischemic stroke. Stroke 2017;48:1203-1209

12. Lee JS, Lee SJ, Hong JM, Choi JW, Hong JH, Chang HW, et al. Temporal changes in care processes and outcomes for endovascular treatment of acute ischemic stroke: retrospective registry data from three Korean centers. Neurointervention 2018;13:212

13. North American Symptomatic Carotid Endarterectomy Trial. Methods, patient characteristics, and progress. Stroke 1991;22:711720

14. Tomsick T, Broderick J, Carrozella J, Khatri P, Hill M, Palesch Y, et al. Revascularization results in the Interventional Management of Stroke II trial. AJNR Am J Neuroradiol 2008;29:582-587

15. Fiorelli M, Bastianello S, von Kummer R, del Zoppo GJ, Larrue V, Lesaffre $\mathrm{E}$, et al. Hemorrhagic transformation within 36 hours of a cerebral infarct: relationships with early clinical deterioration and 3-month outcome in the European Cooperative Acute Stroke Study I (ECASS I) cohort. Stroke 1999;30:2280-2284
16. Frontera JA, Claassen J, Schmidt JM, Wartenberg KE, Temes $\mathrm{R}$, Connolly ES Jr, et al. Prediction of symptomatic vasospasm after subarachnoid hemorrhage: the modified fisher scale. Neurosurgery 2006;59:21-27; discussion 21-27

17. Rha JH, Saver JL. The impact of recanalization on ischemic stroke outcome: a meta-analysis. Stroke 2007;38:967-973

18. Dorado L, Castaño C, Millán M, Aleu A, de la Ossa NP, Gomis M, et al. Hemorrhagic risk of emergent endovascular treatment plus stenting in patients with acute ischemic stroke. J Stroke Cerebrovasc Dis 2013;22:1326-1331

19. Zhu F, Bracard S, Anxionnat R, Derelle AL, Tonnelet R, Liao L, et al. Impact of emergent cervical carotid stenting in tandem occlusion strokes treated by thrombectomy: a review of the TITAN collaboration. Front Neurol 2019;10:206

20. Zhang L, Dai D, Li Z, Duan G, Zhang YW, Yang P, et al. Risk factors for hyperperfusion-induced intracranial hemorrhage after carotid artery stenting in patients with symptomatic severe carotid stenosis evaluation. J Neurointerv Surg 2019;11:474-478

21. Kozar S, Jeromel M. Hyperperfusion and intracranial haemorrhage after carotid angioplasty with stenting-latest review. Signa Vitae 2014;9:9-14

22. Narita S, Aikawa H, Nagata S, Tsutsumi M, Nii K, Yoshida H, et al. Intraprocedural prediction of hemorrhagic cerebral hyperperfusion syndrome after carotid artery stenting. J Stroke Cerebrovasc Dis 2013;22:615-619

23. Abou-Chebl A, Yadav JS, Reginelli JP, Bajzer C, Bhatt D, Krieger DW. Intracranial hemorrhage and hyperperfusion syndrome following carotid artery stenting: risk factors, prevention, and treatment. J Am Coll Cardio/ 2004;43:1596-1601

24. Buhk JH, Cepek L, Knauth M. Hyperacute intracerebral hemorrhage complicating carotid stenting should be distinguished from hyperperfusion syndrome. AJNR Am J Neuroradiol 2006;27:1508-1513

25. Kim BM. Causes and solutions of endovascular treatment failure. J Stroke 2017;19:131-142 